\title{
Possible astrophysical signatures of heavy stable neutral relics in supergravity models
}

\author{
Rouzbeh Allahverdi \\ Physik Department, TU Muenchen, James Frank Strasse, D-85748 Garching, Germany \\ Kari Enqvist \\ Department of Physics and Helsinki Institute of Physics, P.O. Box 9, FIN-00014, University of Helsinki, Helsinki, Finland \\ Anupam Mazumdar \\ The Abdus Salam International Centre for Theoretical Physics, I-34100 Trieste, Italy
}

(Received 28 November 2001; published 14 May 2002)

\begin{abstract}
We consider heavy stable neutral particles in the context of supergravity and show that a gravitationally suppressed inflaton decay can produce such particles in cosmologically interesting abundances within a wide mass range $10^{3} \mathrm{GeV} \leqslant m_{X} \leqslant 10^{11} \mathrm{GeV}$. In gravity-mediated supersymmetry breaking models, a heavy particle can decay into its superpartner and a photon-photino pair or a gravitino. Such decays only change the identity of a possible dark matter candidate. However, for $10^{3} \mathrm{GeV} \leqslant m_{X} \leqslant 10^{7} \mathrm{GeV}$, astrophysical bounds from a gamma-ray background and photodissociation of light elements can be more stringent than the overclosure bound, thus ruling out the particle as a dark matter candidate.

DOI: $10.1103 /$ PhysRevD.65.103519

PACS number(s): 98.80.Cq, 04.65.+e, 12.60.Jv, 95.35.+d
\end{abstract}

\section{INTRODUCTION}

Solving the gauge hierarchy problem via hidden sector supersymmetry breaking at a high scale due to nonperturbative dynamics, which is then mediated to the visible sector through gravity, leads to a phenomenologically successful prediction where sfermions and gauginos get a mass of the order of the electroweak scale [1]. In addition, the superpartner of a graviton, the gravitino, also gets a mass of order 1 $\mathrm{TeV}$ from the super Higgs mechanism [2]. Although the gravitino interactions with matter are suppressed by the Planck scale, they can be generated in a thermal bath from scattering of gauge and gaugino quanta with an abundance given by $[3]^{1}$

$$
\frac{n_{3 / 2}}{s} \approx 10^{-11} \frac{T_{\mathrm{R}}}{10^{11}},
$$

where $s$ defines the entropy density and $T_{R}$ denotes the reheating temperature of the Universe in units of $\mathrm{GeV}$. The gravitinos can then be a source of potential trouble if they decay very late. The decay rate of the gravitino into a gaugino and gauge, or sfermion and fermion quanta, goes as $\Gamma \sim m_{3 / 2}^{3} / M_{p}^{2}$, provided the decay products have negligible

\footnotetext{
${ }^{1}$ Recently, nonthermal production of a helicity $\pm \frac{3}{2}$ gravitino [4] and a helicity $\pm \frac{1}{2}$ gravitino [5] from time-varying inflaton oscillations has been considered. The helicity $\pm \frac{1}{2}$ gravitino for a single chiral multiplet is the superpartner of the inflaton, known as an inflatino. The decay channels of the inflatino have been discussed in Ref. [6]. Also, it has been suggested [6] and explicitly shown [7] that in realistic models with several chiral multiplets, helicity $\pm \frac{1}{2}$ gravitino production is not a problem, so long as the inflationary scale is sufficiently higher than the scale of supersymmetry breaking in the hidden sector and the two sectors are gravitationally coupled.
}

mass compared to the gravitino. This poses a problem for nucleosynthesis for $m_{3 / 2} \sim \mathcal{O}(\mathrm{TeV})$. The gravitinos decay after nucleosynthesis and the decay products can change the abundance of ${ }^{4} \mathrm{He}$ and $\mathrm{D}$ by photodissociation. For $100 \mathrm{GeV} \leqslant m_{3 / 2} \leqslant 1 \mathrm{TeV}$, a successful nucleosynthesis limits the gravitino abundance to be $n_{3 / 2} / s \leqslant\left(10^{-14}-10^{-12}\right)$, which translates to $T_{R} \leqslant\left(10^{7}-10^{9}\right) \mathrm{GeV}$ [8]. This is the simplest illustration of late decaying particles in cosmology, which may release huge entropy while decaying. Various cosmological and astrophysical observations put some useful constraints on the abundance of late decaying particles. There are many other examples within the supergravity inflationary model, which has features similar to gravitinos, such as the moduli fields and the dilaton [9].

Massive particles which decay after nucleosynthesis may also distort the spectrum of the cosmic microwave background or the gamma-ray background. The exact astrophysical signature of such unstable relics depends on their lifetime, thus some part of mass-lifetime parameter space can be ruled out from the present experimental or observational bounds [10].

On the other hand, stable weakly interacting massive particles (WIMPs), denoted here as $X$, generally respect the astrophysical constraints, and can even account for the dark matter in the Universe if they are produced in an interesting abundance. A famous example is the lightest supersymmetric particle (LSP) in supersymmetric extensions of the standard model with unbroken $R$ parity. If LSPs are created in a thermal bath, LSP pairs annihilate to lighter particles when the temperature of the Universe drops below their mass. However, once the annihilation rate drops below the Hubble expansion rate, the LSP comoving number density freezes at its final value. The lower bound on the mass of such species, in order not to overclose the Universe, is a few $\mathrm{GeV}$, the socalled Lee-Weinberg bound [10], while unitarity provides a firm upper bound on their mass $\leqslant 100 \mathrm{TeV}[11]$. 
The above-mentioned bound on the mass of LSP can be evaded if there is no initial thermal equilibrium. In recent years, several mechanisms have been put forward for creating very heavy WIMPs, even superheavy ones with a mass $m_{X}>10^{10} \mathrm{GeV}$, in cosmologically interesting abundances [12-15]. For instance, the production of WIMPs could take place during the phase transition from inflationary to the radiation-dominated or matter-dominated phase [12]. The quantum fluctuations of the field $X$ in a time-varying classical gravitational background may lead to a significant production provided $X$ is stable [12]. This mechanism is largely independent of the nature of $X$ (boson or fermion) and its coupling to other fields. It also works in a variety of inflationary models [15], but abundances close to the dark matter abundance are created for $10^{11} \mathrm{GeV} \leqslant m_{X} \leqslant 10^{13} \mathrm{GeV}$ and $T_{R} \simeq 10^{9} \mathrm{GeV}$.

Another possibility is to create a bosonic field $X$ during the rapid oscillations of the inflaton field from vacuum fluctuations via a coupling $g^{2} \phi^{2} X^{2}$, where $g \sim \mathcal{O}(1)$ [13]. However, one then requires $m_{X} \geqslant 10 m_{\phi}$ and a low reheat temperature, e.g. $T_{R} \sim 10^{2} \mathrm{GeV}$ for $m_{\phi} \sim 10^{13} \mathrm{GeV}$, which makes the mechanism model-dependent.

If the plasma of the inflaton decay products has an instantaneous temperature $T \sim\left(T_{R}^{2} H M_{\text {Planck }}\right)^{1 / 4}$ for $H \geqslant \Gamma_{d}$, where $\Gamma_{d}$ is the inflaton decay rate, it can be much higher than the reheat temperature of the Universe [10]. Then particles of mass $m_{X}>T_{R}$ and with gauge strength interactions can be produced from the annihilation of the relativistic particles in the thermal bath and their abundance freezes at its final value once temperature becomes sufficiently low [14].

However, none of the above-mentioned scenarios for creating WIMPs in the mass range $\sim\left(10^{10}-10^{13}\right) \mathrm{GeV}$ has been actually realized in a supersymmetric setup. For instance, the identity of $X$ is largely unknown apart from the fact that it should be a standard model (SM) gauge singlet. However, if one assumes that $X$ comes from a hidden sector which interacts only gravitationally, they could act as a candidate for dark matter. Within supersymmetry, a heavy particle $X$ is accompanied by its superpartner $\widetilde{X}$ with an almost degenerate mass. This is due to the fact that supersymmetry in the visible sector is broken at a scale $\sim 1 \mathrm{TeV}$. Moreover, in supergravity $X$ and $\widetilde{X}$ have gravitationally suppressed couplings to other fields, among the most notable of which are the inflaton $\phi$, its superpartner inflatino $\widetilde{\phi}$, and the gravitino. This opens up another possibility for creating $X$ via gravitationally suppressed decay of the inflaton. Also, couplings of $X ; \widetilde{X}$ to $\phi ; \widetilde{\phi}$ and the gravitino, together with a small mass difference between $X$ and $\widetilde{X}$, can result in the decay of $X(\widetilde{X})$ into its superpartner and a photon-photino pair, or a gravitino. These decay channels, however, preserve any symmetry which is necessary to forbid $X$ and $\widetilde{X}$ directly decaying into the SM fields. The startling point is that while decaying into its own superpartner, the process changes the identity of a possible dark matter candidate. However, while doing so, the process releases some energy into a cosmic thermal bath. In this paper, we discuss the astrophysical bounds on such decays for gravitino masses ranging from $100 \mathrm{GeV}$ to $1 \mathrm{TeV}$.

\section{PRODUCTION OF $X$ FROM ITS COUPLING TO THE INFLATON SECTOR}

We may consider a complex scalar field $X$ and its fermionic partner $\tilde{X}$ with a superpotential mass term $W \supset(1 / 2) m_{X} \mathbf{X X}$. In the limit of unbroken supersymmetry, $X$ and $\widetilde{X}$ have a common mass $m_{X}$. However, hidden sector supersymmetry breaking generally results in the soft mass term, $m_{3 / 2}^{2}|X|^{2}$, and the $A$ term, $A m_{3 / 2} m_{X} X X+$ H.c. The former elevates the masses of both the components of $X$ above the mass of $\tilde{X}$, while the latter results in the splitting of $X$ into two mass eigenstates $X_{1}$ and $X_{2}$, where we may take $m_{X_{1}}>m_{X_{2}}$. When $m_{X} \gg m_{3 / 2}$, only the contribution of the $A$ term has any significance and yields

$$
m_{\tilde{X}} \approx m_{X}, \quad m_{X_{1}}+m_{X_{2}} \approx 2 m_{X} .
$$

We denote the inflaton multiplet by $\Phi$; it is comprised of the inflaton $\phi$ and the inflatino $\widetilde{\phi}$. Around the global minimum of the potential, the inflation sector superpotential can be approximated as $W \supset(1 / 2) m_{\phi}(\Phi-v)^{2}$, where $v$ is the vacuum expectation value (VEV) of $\phi$ at the minimum. The new inflationary and hybrid inflationary models usually give rise to such a nonzero VEV for either an inflaton or some auxiliary field. There may exist a superpotential term $h_{X} \Phi \mathbf{X X}$ which couples $\Phi$ and $\mathbf{X}$ multiplets. ${ }^{2}$ In general, they can also be coupled via higher-dimensional Plancksuppressed terms. For example, consider the minimal supergravity where the scalar potential is given by [1]

$$
V=e^{G}\left(\frac{\partial G}{\partial \varphi_{i}} \frac{\partial G}{\partial \varphi_{i}^{*}}-\frac{3}{M_{\text {Planck }}^{2}}\right) .
$$

Here $G$ is the Kähler function defined by

$$
G=\frac{\varphi_{i} \varphi_{i}^{*}}{M_{\text {Planck }}^{2}}+\ln \left(\frac{|W|^{2}}{M_{\text {Planck }}^{6}}\right),
$$

where $\varphi_{i}$ are scalar fields in the theory. There also exists a term in the Lagrangian,

$$
e^{G / 2}\left(\frac{\partial G}{\partial \varphi_{i}} \frac{\partial G}{\partial \varphi_{j}}-\frac{\partial^{2} G}{\partial \varphi_{i} \partial \varphi_{j}}\right) \tilde{\tilde{\varphi}}_{i} \tilde{\varphi}_{j}+\text { H.c. },
$$

with $\tilde{\varphi}_{i}$ being the fermionic partner of $\varphi_{i}$. For our choice of superpotential, the following terms can then be identified in the Lagrangian:

$$
\frac{v m_{X}}{M_{\text {Planck }}^{2}} \phi \widetilde{X} \widetilde{X}, \quad \frac{v m_{X}}{M_{\text {Planck }}^{2}} \widetilde{\phi} \widetilde{X} X, \quad \frac{v m_{\phi} m_{X}}{M_{\text {Planck }}^{2}} \phi X X
$$

and the coupling $h_{X}$ is recognized as

\footnotetext{
${ }^{2}$ Note that a superpotential term $\mathbf{X} \Phi \Phi$, which is linear in $\mathbf{X}$, provides a decay channel for $X$ and $\widetilde{X}$ thus ignored in our discussion.
} 


$$
h_{X}=\frac{v m_{X}}{M_{\text {Planck }}^{2}} .
$$

\section{A. Production by direct inflaton decay}

The terms in Eq. (6) result in $X(\widetilde{X})$ production from inflaton decay if $m_{X}<m_{\phi} / 2$, with a rate $\Gamma_{X} \sim h_{X}^{2} m_{\phi} / 8 \pi$. The total inflaton decay rate is given by $\Gamma_{d} \sim \sqrt{8 \pi / 3} T_{R}^{2} / M_{\text {Planck }}$, while the inflaton number density at the time of decay is given by $n_{\phi} \sim T_{R}^{4} / m_{\phi}$. This constrains the overall coupling to

$$
h_{X}^{2} \leqslant 32 \pi \sqrt{\frac{8 \pi}{3}} \frac{T_{R}}{M_{\text {Planck }}} \frac{10^{-9}}{m_{X}},
$$

where $m_{X}$ is in units of $\mathrm{GeV}$. This is required due to the fact that the produced $X(\tilde{X})$ must not overclose the Universe which, for $\Omega_{X} \leqslant 0.3$ and $H_{0}=70 \mathrm{~km} \mathrm{sec}^{-1} \mathrm{Mpc}^{-1}$, reads

$$
\frac{n_{X}}{n_{\gamma}} \leqslant \frac{4 \times 10^{-9}}{m_{X}} \text {, }
$$

when $m_{X}$ is expressed in units of GeV. In the particular case in which $\Phi$ and $\mathbf{X}$ multiplets are gravitationally coupled, one finds

$$
m_{X}^{3}\left(\frac{v}{M_{\text {Planck }}}\right)^{2} \leqslant 10^{-7} M_{\text {Planck }} T_{R}
$$

If $v \simeq M_{\text {Planck }}$, then $X(\tilde{X})$ within the mass range $10^{3} \mathrm{GeV}$ $\leqslant m_{X} \leqslant 10^{7} \mathrm{GeV}$ can be produced in interesting abundances for a range of reheat temperatures $1 \mathrm{MeV} \leqslant T_{R} \leqslant 10^{10} \mathrm{GeV}$.

If the inflaton VEV is lowered down to $v / M_{\text {Planck }} \simeq 10^{-6}$, then $X(\tilde{X})$ with a mass range $10^{7} \mathrm{GeV} \leqslant m_{X} \leqslant 10^{11} \mathrm{GeV}$ can become a dark matter candidate for the same range of reheat temperature. $^{3}$

\section{B. Production from the thermal bath}

Another possibility for production of $X$ is from its indirect coupling to the thermal bath via the inflaton sector. The simplest situation will be for the case in which inflaton is coupled to two fermions (hence the inflatino is coupled to a fermion-boson pair). As an example, consider the $\phi \tilde{\gamma} \tilde{\gamma}$ and $\widetilde{\phi} \tilde{\gamma} \gamma$ couplings. ${ }^{4}$ These couplings are supersymmetric partners and the latter one is responsible for a Hubble-induced gaugino mass term $\propto H \tilde{\gamma} \tilde{\gamma}$. Then $X(\tilde{X})$ can be produced in a thermal bath from the $s$-channel diagram which includes

\footnotetext{
${ }^{3}$ It is evident from the overclosure bound that $h_{X}$ needs to be very small. This may look $a d$ hoc for a superpotential coupling between $\Phi$ and $\mathbf{X}$, while a nonrenormalizable gravitationally suppressed coupling in the context of supergravity offers a natural explanation for such a small coupling if $X$ belongs to a hidden sector.

${ }^{4}$ The situation when $\gamma$ and $\tilde{\gamma}$ are replaced by a light particle and its superpartner is similar.
}

$h \phi \tilde{\gamma} \tilde{\gamma}(h \widetilde{\phi} \tilde{\gamma} \gamma)$ and $h_{X} \phi \tilde{X} \tilde{X}\left(h_{X} \widetilde{\phi} \widetilde{X} X\right)$ couplings at the first and second vertices, respectively. Assuming that $m_{X} \leqslant T_{R}$, the rate for $X(\tilde{X})$ production is given by

$$
\Gamma_{X} \sim \frac{h^{2} h_{X}^{2}}{16 \pi^{2} m_{\phi}^{2}} T_{R}^{3},
$$

where $m_{\phi} \approx m_{\tilde{\phi}}$, since hidden sector supersymmtery breaking only results in $\left|m_{\phi}-m_{\tilde{\phi}}\right| \ll m_{\phi}$. The coupling $h$ obeys the following relationship:

$$
h^{2} \frac{m_{\phi}}{8 \pi} \sim \sqrt{\frac{8 \pi}{3}} \frac{T_{R}^{2}}{M_{\text {Planck }}},
$$

and the number density of created $X(\widetilde{X})$ particles is as follows:

$$
\frac{n_{X}}{n_{\gamma}} \sim \frac{h_{X}^{2}}{2 \pi}\left(\frac{T_{R}}{m_{\phi}}\right)^{3} .
$$

Here the main contribution to $X(\tilde{X})$ production occurs at $H$ $\simeq \Gamma_{d}$. In order for $X(\tilde{X})$ not to overclose the Universe, it is necessary to have $\Omega_{X} \leqslant 0.3$, which implies

$$
h_{X}^{2} \leqslant \frac{3 \times 10^{-8}}{m_{X}}\left(\frac{m_{\phi}}{T_{R}}\right)^{3} .
$$

We always have $\Gamma_{X}<H$ since $h_{X}^{2} T_{R}^{3}<m_{\phi}^{3}$ (note that for perturbative inflaton decay $\left.T_{R}<m_{\phi}\right)$. This ensures that $X(\tilde{X})$ will never reach equilibrium with the thermal bath. However, the bound on $h_{X}$ from (8) is much stronger than the bound in (14). This implies that $X(\widetilde{X})$ production from the thermal bath is always subleading with respect to production by direct inflaton decay in order for the latter not to saturate the overclosure bound.

It is interesting that direct inflaton decay in supergravity may produce $X(\tilde{X})$ in a wide range of mass with a cosmologically interesting abundance. This is particularly significant as some of the proposed mechanism for creating heavy WIMPs might not work in a supersymmetric setup. For example, it is known that scalar fields, and also fermions if allowed by symmetry considerations, acquire a Hubbleinduced mass term during and after inflation in supergravity models. This suggests that production of such particles from a time-varying gravitational backgrouned might not be possible at all unless such Hubble-induced terms are small, or canceled for a particular choice of superpotential.

\section{VERY LATE DECAY OF $X(\widetilde{X})$}

There exist strong astrophysical bounds on the late decay of particles to photons or charged particles. For a detailed study of various constraints on such decays, we refer the reader to Refs. $[16,17]$. Here we briefly mention the relevant bounds. For a late decay such as $10^{5} \mathrm{~s} \leqslant \tau \leqslant 10^{13} \mathrm{~s}$, where $\tau$ denotes the lifetime of a particle, the decay products can alter the chemical potential of the microwave background pho- 
tons. Note that recombination occurs at $10^{13} \mathrm{~s}$. The reason is that photon number changing interactions such as $e \gamma$ $\rightarrow e \gamma \gamma$ go out of equilibrium at $t>10^{5} \mathrm{~s}$, thus inducing an effective chemical potential for the microwave background photons. The chemical potential has been given in [18] for the case in which decay photons thermalize instantly and the current experimental bound is [19]

$$
\mu=\frac{\rho_{d}}{\rho_{\gamma}} \leqslant 3.3 \times 10^{-4},
$$

where $\rho_{d}$ and $\rho_{\gamma}$ denote the energy density in the decay and background photons, respectively. If the decay occurs after recombination and before the present era, then the nonthermal decay photons may be directly visible today if the optical depth back to the decay epoch is small enough. Then the main astrophysical constraint is that the flux of decay photons must not exceed that of the observed differential photon flux, which is given by $[10]^{5}$

$$
\mathcal{F}_{\gamma}(E) \leqslant \frac{\mathrm{MeV}}{E} \mathrm{~cm}^{-2} \mathrm{sr}^{-1} \mathrm{~s}^{-1}
$$

where $E$ is the photon energy and the observed photon flux is denoted by $\mathcal{F}_{\gamma}$.

There are also bounds on late decay to photons coming from nucleosynthesis. If $\tau \geqslant 10^{4} \mathrm{~s}$, the photonic showers can change the abundance of light elements. ${ }^{6}$ For $10^{4} \mathrm{~s} \leqslant \tau$ $\leqslant 10^{6} \mathrm{~s}$, photodestruction of $D$ will reduce its abundance, while for $\tau>10^{6} \mathrm{~s}$, photoproduction of $D$ and ${ }^{3} \mathrm{He}$ from the destruction of ${ }^{4} \mathrm{He}$ will give the main constraint.

\section{A. $X(\widetilde{X}) \rightarrow \widetilde{X}(X)+\gamma+\tilde{\gamma}$ via off-shell gravitino}

Henceforth, we shall consider the case in which the $\mathbf{X}$ multiplet has only gravitationally suppressed couplings to the matter sector. ${ }^{7}$ Depending on the mass differences among scalar and fermionic components of $X$, different situations may arise. If $\Delta m \equiv\left|m_{X_{1,2}}-m_{\tilde{X}}\right|>m_{3 / 2}$, the decay channels $X_{1} \rightarrow \widetilde{X}+\widetilde{G}$ and $\widetilde{X} \rightarrow X_{2}+\widetilde{G}$ are kinematically allowed. For

\footnotetext{
${ }^{5}$ Decay photons generally trigger an electromagnetic shower, since their scattering off the microwave background photons can create $e^{+} e^{-}$pairs, which themselves undergo Compton scattering off photons. Moreover, $\gamma-\gamma$ scattering redistributes photon energy. Therefore, one must take these effects into account in order to compare with the observed photon flux, as done in $[16,17]$.

${ }^{6}$ The $\gamma-\gamma$ scattering dominates photon interactions for $t<10^{4} \mathrm{~s}$ and hence photonic showers will not affect light element abundances.

${ }^{7}$ In the case in which $X(\tilde{X})$ has common gauge interactions with matter fields, the three-body decays $X_{1}(\tilde{X}) \rightarrow \tilde{X}\left(X_{2}\right)+\gamma+\tilde{\gamma}$ can occur via gauge interactions [in fact through the same diagrams, which results in the production of $X(\widetilde{X})$ in a thermal bath]. In this case, the decay rate is quite large, $\Gamma \sim \alpha^{2} \Delta m^{3} / m_{X}^{2}$; notice that there is a phase-space suppression, and the decay occurs before nucleosynthesis. Again note that the $X_{2}$ component is the strictly stable particle.
}

$\Delta m<m_{3 / 2}$ and $\Delta m>m_{\mathrm{LSP}}$, provided the gravitino is not the LSP, the three-body decay $X_{1} \rightarrow \widetilde{X}+\gamma+\tilde{\gamma}$ (and correspondingly $\widetilde{X} \rightarrow X_{2}+\gamma+\tilde{\gamma}$ ) can occur. We indeed expect that such decays take place since $\Delta m \ll m_{X}$ implies that any production mechanism shall produce $X_{1}, X_{2}$, and $\widetilde{X}$ (at least $X_{1}$ and $X_{2}$ ) in comparable abundances. Moreover, we notice that in both cases the $X_{2}$ component is the strictly stable particle and hence a dark matter candidate. Then its abundance, which is the same as the initial abundance of $X(\widetilde{X})$, must not saturate the overclosure bound in (9).

Let us first consider the case in which $\Delta m<m_{3 / 2}$. This is the situation in the hidden sector supersymmetry breaking scenario with the Polonyi field, where $\Delta m \approx(1-\sqrt{3} / 2) m_{3 / 2}$ [20]. As long as $\Delta m>m_{\mathrm{LSP}}$, the three-body decay $X(\tilde{X})$ $\rightarrow \widetilde{X}(X)+\gamma+\tilde{\gamma}$, via an off-shell gravitino, is kinematically allowed. The decay diagram includes two vertices: at the first vertex, $X$ and $\widetilde{X}$ couple to $\widetilde{G}$ while the gravitino is coupled to a $\gamma \tilde{\gamma}$ pair at the second vertex. The decay rate is doubly Planck-mass-suppressed, and with phase-space suppression leads to

$$
\Gamma_{1} \sim \frac{1}{32(2 \pi)^{3}} \times \frac{m_{X}^{2}\left(\Delta m-m_{\mathrm{LSP}}\right)^{3}}{M_{p}^{4}} .
$$

Here we have assumed that the decay matrix element is constant over the phase space. An interesting observation is that the decay rate is model-independent except for the appearance of $\Delta m$. We make the resonable assumption that $100 \mathrm{GeV} \leqslant \Delta m-m_{\mathrm{LSP}} \leqslant 800 \mathrm{GeV}$ for $m_{3 / 2} \simeq 1 \mathrm{TeV}$. Then the lifetime $\tau \sim \Gamma_{1}^{-1}$ can be of the same order as the age of the Universe $\simeq 10^{17} \mathrm{~s}$ for the mass range

$$
10^{13} \mathrm{GeV}<m_{X}<10^{15} \mathrm{GeV} .
$$

For a lighter $m_{X}$, the lifetime is longer than the age of the Universe.

The decay usually releases entropy, which is worth estimating in our case. Since $\Delta m-m_{\mathrm{LSP}} \ll m_{X}$, the released momentum is distributed among all three decay products while the energy in the mass difference is practically carried by $\gamma$ and $\tilde{\gamma}$. It is therefore reasonable to consider the energy which is taken away by $\gamma$ to be $\left(\Delta m-m_{\mathrm{LSP}}\right) / 2$. Then the released entropy is $s_{d} \sim\left[(50-400) n_{X}\right]^{3 / 4}$ while the entropy in the cosmic microwave background photons is $s \sim n_{\gamma}$, resulting in

$$
\frac{s_{d}}{s} \leqslant\left(\frac{4 \times 10^{-7}}{T_{\gamma} m_{X}}\right)^{3 / 4},
$$

where we have used the overclosure bound. Here $T_{\gamma}$ denotes the temperature of the background photons at the time of decay and has its smallest value $\sim 5 \times 10^{-13} \mathrm{GeV}$ for the decays occurring at the present moment. This implies that for $m_{X} \leqslant 10^{6} \mathrm{GeV}$, the decay products increase the entropy of the Universe at the time of decay. However, in this case the decay occurs much later than today and hence entropy release will not be substantial at the present epoch. Therefore, 
the three-body decay does not release any significant entropy within the lifetime of the Universe.

Nevertheless, the observed photon flux puts a severe constraint on the abundance of $X$. For a decay energy of order $(50-400) \mathrm{GeV}$, the most stringent limit is $[16,17]$

$$
\frac{n_{X}}{n_{\gamma}} \leqslant 10^{-19},
$$

while for a wide range of decay lifetimes $10^{17} \mathrm{~s} \leqslant \tau$ $\leqslant 10^{21} \mathrm{~s}$, the bound is given by $[16,17]$

$$
\frac{n_{X}}{n_{\gamma}} \leqslant\left(10^{-18}-10^{-13}\right)
$$

The most conservative mass range which leads to a decay lifetime in this range is $10^{11} \mathrm{GeV} \leqslant m_{X} \leqslant 10^{17} \mathrm{GeV}$. The overclosure limit now reads $n_{X} / n_{\gamma} \leqslant 4 \times 10^{-20}$, which is more stringent by several orders of magnitude. Therefore, the three-body decay satisfies bounds on photon flux if $\Omega_{X}$ $\leqslant 0.3$.

If $X$ is very massive it will also be possible that the decay occurs before recombination. For example, if $m_{X}$ $\sim 10^{16.5} \mathrm{GeV}$, which can be related to the string scale in $M$ theory, and $\Delta m-m_{\mathrm{LSP}} \simeq 1 \mathrm{TeV}$, the process $X \rightarrow \tilde{X}+\gamma+\tilde{\gamma}$ may take place after the photon number changing interactions $e \gamma \rightarrow e \gamma \gamma$ have gone out of equilibrium, but before the recombination era. For an energy release of order (50 $-400) \mathrm{GeV}$, in order to satisfy the present constraint on the chemical potential, from Eq. (15), we obtain the bound $n_{X} / n_{\gamma} \leqslant 10^{-14}$. This is again much weaker than the overclosure bound $n_{X} / n_{\gamma} \leqslant 4 \times 10^{-25}$. We therefore conclude that avoiding the overclosure of the Universe puts a stronger bound on the abundance of $X$ than astrophysical constraints from the decay process $X(\tilde{X}) \rightarrow \widetilde{X}(X)+\gamma+\tilde{\gamma}$, via an offshell gravitino.

\section{B. $X(\widetilde{X}) \rightarrow \widetilde{X}(X)+\gamma+\tilde{\gamma}$ via off-shell inflatino}

Now, we turn our attention to another possible decay channel where the three-body decay of $X(\tilde{X})$ can occur via an off-shell inflatino. The decay diagram will include the $h_{X} X \tilde{X} \widetilde{\phi}$ coupling at the first vertex while at the second vertex $\widetilde{\phi}$ is coupled to a $\gamma \tilde{\gamma}$ pair (as discussed in the previous section). The significance of $X$ decay via an off-shell inflatino now depends on the dominant decay channel of an inflaton (inflatino). If the inflaton (inflatino) dominantly decays to a three-body final state, we will have a four-body $X$ decay. This is even more phase-space-suppressed (and perhaps forbidden) than the three-body decay via an off-shell gravitino. On the other hand, if the inflaton (inflatino) mainly decays to a two-body final state, in particular to a $\gamma \tilde{\gamma}$ pair, we may have a three-body decay of $X$ via the inflatino. Then the rate for the decay $X(\tilde{X}) \rightarrow \widetilde{X}(X)+\gamma+\tilde{\gamma}$ is found to be

$$
\Gamma_{2} \sim 10^{-2} h_{X}^{2} \frac{T_{R}^{2} M_{\text {Planck }}}{m_{\phi}^{3}} \frac{\left(\Delta m-m_{\text {LSP }}\right)^{3}}{M_{\text {Planck }}^{2}} .
$$

As pointed out earlier, the coupling $h_{X}$ must satisfy the inequality in (8) in order for $X$ not to overclose the Universe if $m_{X}<m_{\phi} / 2$. This yields

$$
\Gamma_{2} \sim \frac{4 \times 10^{-9}}{m_{X}}\left(\frac{T_{R}}{m_{\phi}}\right)^{3} \frac{\left(\Delta m-m_{\mathrm{LSP}}\right)^{3}}{M_{\text {Planck }}^{2}} .
$$

Let us first consider the case with a decay energy of order $400 \mathrm{GeV}$. Then for $m_{X}=10^{5}\left(10^{10}\right) \mathrm{GeV}$ the decay lifetime is $\tau_{2} \sim 10^{17}\left(10^{22}\right) \mathrm{s}$, provided $T_{R} \simeq m_{\phi}$. The gamma-ray background and the overclosure bounds then translate to $n_{X} / n_{\gamma} \leqslant 10^{-17}\left(10^{-12}\right) \quad[16,17]$ and $n_{X} / n_{\gamma} \leqslant 10^{-14}\left(10^{-19}\right)$, respectively. For masses $10^{5} \mathrm{GeV} \leqslant m_{X} \leqslant 10^{7} \mathrm{GeV}$, the gamma-ray bound is indeed stronger, thus providing a clear signal that within this mass range $X$ cannot act as a stable dark matter candidate. For $T_{R} / m_{\phi} \simeq 10^{-1}$, astrophysical constraints turn out to be more stringent than the overclosure bound within the mass range $10^{3} \mathrm{GeV} \leqslant m_{X} \leqslant 10^{5} \mathrm{GeV}$, while for a smaller $T_{R} / m_{\phi}$, the main constraint appears from the overclosure bound. For example, for $T_{R} / m_{\phi}$ $\leqslant 10^{-2}$, the overclosure bound ensures that for $m_{X}$ $\geqslant 1 \mathrm{TeV}$ the three-body decay will not be astrophysically dangerous.

For a decay energy of order $50 \mathrm{GeV}$, a mass $m_{X}$ $=10^{3}\left(10^{7}\right) \mathrm{GeV}$ results in the decay lifetime $\tau_{2}$ $\sim 10^{18}\left(10^{22}\right) \mathrm{s}$, provided $T_{R} \simeq m_{\phi}$. The gamma-ray background and the overclosure bounds yield $n_{X} / n_{\gamma}$ $\leqslant 10^{-18}\left(10^{-13}\right)[16,17]$ and $n_{X} / n_{\gamma} \leqslant 10^{-12}\left(10^{-16}\right)$, respectively, where for $10^{3} \mathrm{GeV} \leqslant m_{X} \leqslant 10^{5} \mathrm{GeV}$ the gamma-ray bound will be stronger. For $T_{R} / m_{\phi} \simeq 10^{-1}$, astrophysical constraints are more stringent within a narrow mass range $10^{3} \mathrm{GeV} \leqslant m_{X} \leqslant 10^{4} \mathrm{GeV}$, while for a smaller $T_{R} / m_{\phi}$ the main constraint appears from the overclosure bound.

If we desire to have a supermassive $X$ with a mass $m_{X}$ $>m_{\phi} / 2$, the production of $X$ through direct decay of the inflaton cannot be possible. Therefore, even if such massive $X$ might have been created, their decay would follow Eq. (22) and could easily occur before recombination. However, mechanisms which could create $X$ in this case prefer a mass $m_{X}>10^{10} \mathrm{GeV}[12,13,15]$. The overclosure bound then yields $n_{X} / n_{\gamma} \leqslant 10^{-19}$, which for an energy release 50 $-400 \mathrm{GeV}$ in the three-body decay is always stronger than the astrophysical constraints.

Our overall conclusion is that for $m_{3 / 2} \simeq 1 \mathrm{TeV}$, the decay process $X(\widetilde{X}) \rightarrow \widetilde{X}(X)+\gamma+\tilde{\gamma}$ can be astrophysically dangerous when mediated by an inflatino. In particular, the astrophysical bounds are stronger than the overclosure bound for the mass range $10^{3} \mathrm{GeV} \leqslant m_{X} \leqslant 10^{7} \mathrm{GeV}$ when $10^{-1}$ $\leqslant T_{R} / m_{\phi} \leqslant 1$.

$$
\text { C. } X(\widetilde{X}) \rightarrow \widetilde{X}(X)+\widetilde{G}
$$

Let us finish by discussing the two-body decay case which occurs when $\Delta m>m_{3 / 2}$. This is also quite plausible since, as pointed out in Ref. [20], for a generic supersymmetry breaking scenario one may not have $\Delta m<m_{3 / 2}$. The decay rate for the process $X(\widetilde{X}) \rightarrow \widetilde{X}(X)+\widetilde{G}$ is, however, Planck-mass-suppressed, and phase-space suppression gives 


$$
\Gamma_{3} \sim \frac{m_{X}^{2} m_{3 / 2}}{8 \pi M_{\text {Planck }}^{2}}
$$

As we have mentioned earlier, in such a case the most important bounds come from the success of big-bang nucleosynthesis. Here the main danger arises from the produced gravitinos with a mass $m_{3 / 2}=100 \mathrm{GeV}$, which decay into energetic photons thus constraining their abundance to $n_{X} / n_{\gamma} \leqslant 10^{-14}$ [8] (here we obviously assume that the gravitino is not the LSP). This requires that $m_{X} \geqslant 10^{6} \mathrm{GeV}$ if $X$ is produced with an abundance $\Omega_{X} \sim 0.3$. On the other hand, for a gravitino mass $m_{3 / 2}=1 \mathrm{TeV}$, the nucleosynthesis bound results in $n_{X} / n_{\gamma} \leqslant 10^{-12}$ [8], which relaxes the mass range of $X$, and masses beyond $10 \mathrm{TeV}$ could easily be accommodated.

Some comments are in order. The decay of a particle into its superpartner and a gravitino can in general occur for unstable species as well. However, in such cases the suppression of this decay mode relative to other decay channels ensures that nucleosynthesis will not be disrupted (e.g., the case for inflaton decay has been considered in Ref. [20]). On the other hand, for a particle which is stable in the limit of unbroken supersymmetry, this decay mode (and the threebody decays discussed earlier) are the only possible channels. We also limited our discussion to gravity-mediated models of supersymmtery breaking. The reason is that in gauge-mediated models the gravitino mass is substantially smaller than the weak scale, implying that the gravitino is the LSP. Then a possible two-body decay does not pose a threat to nucleosynthesis, while three-body decays are kinematically forbidden. Finally, we only considered neutral stable particles in this paper. For a charged particle, the dominant decay mode will be $X(\tilde{X}) \rightarrow \widetilde{X}(X)+\tilde{\gamma}$, which occurs much before nucleosynthesis. Moreover, the abundance of such particles is severely constrained by their searches in the sea water, which is much more stringent than the overclosure bound [21].

\section{CONCLUSION}

We have discussed the possible astrophysical signatures of a neutral stable particle within the context of supergravity. We considered a multiplet $\mathbf{X}$ which has a mass $m_{X}$ and is stable in the limit of unbroken supersymmtery. Supersymmetry breaking in the hidden sector generally results in the pattern $m_{X_{1}}>m_{\tilde{X}}>m_{X_{2}}$, so long as $m_{X} \gg m_{3 / 2}$, where $X_{1}, X_{2}$, and $\tilde{X}$ are the two scalar components and the fermionic component of the multiplet, respectively. We have noticed that a gravitationally suppressed inflaton decay could lead to production of $X(\widetilde{X})$ in interesting abundances in a wide mass range, $10^{3} \mathrm{GeV} \leqslant 10^{11} \mathrm{GeV}$. Details depend on the inflaton $\mathrm{VEV}$ at the minimum and the reheat temperature of the Universe, which we always assume to be smaller than $10^{10} \mathrm{GeV}$, in order to avoid thermal gravitino overproduction.

We also considered the decay of $X(\widetilde{X})$ into its superpartner and a photon-photino pair, or a gravitino, and we discussed the various astrophysical and overclosure bounds which restrict the decay channels. If $X(\widetilde{X})$ is produced with an abundance large enough to account for the dark matter in the Universe, such decays merely change the identity of the dark matter candidate. Depending on the supersymmetry breaking scenario, the mass difference between $X$ and $\tilde{X}$ can be smaller or larger than the mass of the gravitino. In the former case, the two-body decay channels are kinematically forbidden. However, the three-body decay via an off-shell gravitino and/or an off-shell inflatino may still occur. The three-body decays release an energy which is just the mass difference between $X, \tilde{X}$, and LSP. In the case in which $X(\tilde{X})$ decays into $\widetilde{X}(X)$ and a photon-photino pair, via an off-shell gravitino, for the mass range $10^{11} \mathrm{GeV} \leqslant m_{X} \leqslant 10^{17} \mathrm{GeV}$, the main constraint comes from the overclosure bound yielding $n_{X} / n_{\gamma} \leqslant 10^{-20}$. For $X(\widetilde{X})$ decaying via an off-shell inflatino, the situation depends on a number of model parameters such as the reheat temperature and the mass of the inflaton.

We also pointed out that for larger ratios of $T_{R} / m_{\phi}$, the gamma-ray background could in principle constrain the lower half of the mass range $10^{3} \mathrm{GeV} \leqslant m_{X} \leqslant 10^{7} \mathrm{GeV}$, while the upper half is constrained by the overclosure limit. We also considered the decay of $X(\tilde{X})$ to $\widetilde{X}(X)$ and a gravitino when the mass difference between them is larger than the gravitino mass. In this case, the main constraint comes from avoiding the photodissociation of light elements. Indeed, the abundance of $X$ must be smaller than $10^{-14}$ for $m_{3 / 2}=100 \mathrm{GeV}$ implying that $m_{X} \geqslant 10^{6} \mathrm{GeV}$ if $X$ is the dark matter in the Universe.

\section{ACKNOWLEDGMENTS}

The authors are thankful to A. Özpineci, A. PerezLorenzana, and S. Sarkar for valuable discussions. R.A. is supported by "Sonder-forchschungsberich 375 für AstroTeilchenphysik" der Deutschen Forschungsgemeinschaft, K.E. is supported partly by the Academy of Finland under Contract No. 101-35224, and A.M. acknowledges the support of The Early Universe Network HPRN-CT-2000-00152 and the hospitality of the Helsinki Institute of Physics, where part of the work has been carried out.
[1] P. Nath, R Arnowitt, and A. Chamseddine, Applied N=1 Supergravity (World Scientific, Singapore, 1984); R. Barbieri, S. Ferrara, and C.A. Savoy, Phys. Lett. 119B, 343 (1982); A. Chamseddine, R. Arnowitt, and P. Nath, Phys. Rev. Lett. 49,
970 (1982); H.P. Nilles, M. Srednicki, and D. Wyler, Phys. Lett. 120B, 346 (1983); L. Alvarez-Gaumez, J. Polchinski, and M. Wise, Nucl. Phys. B221, 495 (1983).

[2] S. Deser and B. Zumino, Phys. Rev. Lett. 38, 1433 (1977). 
[3] J. Ellis, J.E. Kim, and D.V. Nanopoulos, Phys. Lett. 145B, 181 (1984); J. Ellis, D.V. Nanopoulos, K.A. Olive, and S-J. Rey, Astropart. Phys. 4, 371 (1996); for a recent calculation, see M. Boltz, A. Brandenburg, and W. Buchmuller, Nucl. Phys. B606, 518 (2001).

[4] A.L. Maroto and A. Mazumdar, Phys. Rev. Lett. 84, 1655 (2000).

[5] R. Kallosh, L. Kofman, A. Linde, and A. Von Proeyen, Phys. Rev. D 61, 103503 (2000); G.F. Giudice, I. Tkachev, and A. Riotto, J. High Energy Phys. 08, 009 (1999).

[6] R. Allahverdi, M. Bastero-Gil, and A. Mazumdar, Phys. Rev. D 64, 023516 (2001).

[7] H.P. Nilles, M. Peloso, and L. Sorbo, Phys. Rev. Lett. 87, 051302 (2001); J. High Energy Phys. 04, 004 (2001).

[8] M. Kawasaki, K. Kohri, and T. Moroi, Phys. Rev. D 63, 103502 (2001); for a review, see S. Sarkar, Rep. Prog. Phys. 59, 1493 (1996).

[9] G.D. Coughlan, W. Fischler, E.W. Kolb, S. Raby, and G.G. Ross, Phys. Lett. 131B, 53 (1983); J. Ellis, D.V. Nanopoulos, and M. Quiros, Phys. Lett. B 174, 176 (1986); B. de Carlos, J.A. Casas, F. Quevedo, and E. Roulet, ibid. 318, 447 (1993).

[10] E.W. Kolb and M.S. Turner, The Early Universe (Addison
Wesley, New York, 1990).

[11] K. Griest and M. Kamionkowski, Phys. Rev. Lett. 64, 615 (1990).

[12] D.J.H. Chung, E.W. Kolb, and A. Riotto, Phys. Rev. D 59, 023501 (1999).

[13] D.J.H. Chung, E.W. Kolb, and A. Riotto, Phys. Rev. Lett. 81, 4048 (1998).

[14] D.J.H. Chung, E.W. Kolb, and A. Riotto, Phys. Rev. D 60, 063504 (1999)

[15] D.J.H. Chung, P. Crotty, E.W. Kolb, and A. Riotto, Phys. Rev. D 64, 043503 (2001).

[16] J. Ellis, G.B. Gelmini, J.L. Lopez, D.V. Nanopoulos and S. Sarkar, Nucl. Phys. B373, 399 (1992).

[17] G. Kribs and I. Rothstein, Phys. Rev. D 55, 4435 (1997); 56, 1822(E) (1997).

[18] A.D. Dolgov and Ya.B. Zeldovich, Rev. Mod. Phys. 53, 1 (1981); P.J.E. Peebles, Physical Cosmology (Princeton University Press, Princeton, NJ, 1971).

[19] J.C. Mather et al., Astrophys. J. 420, 439 (1994).

[20] H.P. Nilles, K.A. Olive, and M. Peloso, Phys. Lett. B 522, 304 (2001).

[21] A. Kudo and M. Yamaguchi, Phys. Lett. B 516, 151 (2001). 